\title{
IRIS: a novel approach to monitoring risk in safety critical plants
}

\author{
Elisa Calá \\ IB s.r.l., Rapallo GE, Italy. E-mail: e.cala@ib-industry.com
}

Guglielmo Carrubba

SAPIO Produzione Idrogeno Ossigeno s.r.l., Monza, Italy. E-mail: guglielmo.carrubba@sapio.it

\author{
Michele Compare \\ Energy Department, Politecnico di Milano, Italy.E-mail: michele.compare@polimi.it \\ Aramis s.r.l., Milano, Italy. E-mail: michele.compare@aramis3d.com
}

\author{
Alessio Martini \\ IB s.r.l., Rapallo GE, Italy.E-mail: a.martini@ib-industry.com
}

Roberto Mascherona

Aramis s.r.l., Milano, Italy. E-mail: roberto.Mascherona@aramis3d.com

Maurizio Ricci

IB s.r.l., Rapallo GE, Italy.E-mail: m.ricci@ib-industry.com

Enrico Zio

Energy Department, Politecnico di Milano, Italy.E-mail: enrico.zio@polimi.it

Aramis s.r.l., Milano, Italy

MINES ParisTech, PSL Research University, CRC, Sophia Antipolis, France

Eminent Scholar, Department of Nuclear Engineering, College of Engineering, Kyung Hee University,

Republic of Korea

We present a novel approach for tracking a global risk index of a safety-critical industrial plant. This approach, called Integrated Risk Index by Sapio (IRIS), considers safety as a critical process variable and relies on a combination of techniques such as Bayesian Networks, Multi Attribute Value Theory, Cognitive Reliability and Error Analysis Method. The approach is described through a case study of the cryogenic gas production industry.

Keywords: Global Risk Index, Bayesian Networks, MAVT, CREAM, Cryogenic gas.

\section{Introduction}

Industry 4.0, the fourth industrial revolution, aims at creating smart factories, equipped with disruptive technologies such as advanced robotics, high computing power and connectivity, etc., which are integrated with analytical and cognitive technologies that enable machine-to-machine (M2M) and machine-to-human (M2H) communication Hermann et al. (2016); Drath and Horch (2014). One of the opportunities of Industry 4.0 is Predictive Maintenance (PdM), which makes use of condition monitoring data for detecting, diagnosing and prognosing anomalies to dynamically manage maintenance. The industrial-scale deployment of PdM involves many other aspects, including safety. Intuitively, data collected on the system from appropriately installed sensors can be used for risk monitoring aimed at promptly actuating informed responses to incidents and accidents.

Only few experiences of condition monitoring for safety applications have been proposed (e.g., Coble et al. (2015); Daigle et al. (2017); Zio (2018); Di Maio et al. (2018)), and a structured modeling approach that considers risk as a monitored process variable in safety-critical industrial contexts is still not available, to the authors' best knowledge.

In this paper, we develop the Integrated Risk Index by Sapio (IRIS) innovative approach that relies on condition monitoring data, and operation and maintenance information for tracking a global risk index of a safety-critical plant. This approach emerges from an original idea by SAPIO, which

Proceedings of the 30th European Safety and Reliability Conference and

the 15th Probabilistic Safety Assessment and Management Conference

Edited by Piero Baraldi, Francesco Di Maio and Enrico Zio

Copyright (C) ESREL2020-PSAM15 Organizers.Published by Research Publishing, Singapore.

ISBN: 978-981-14-8593-0; doi:10.3850/978-981-14-8593-0 
has taken shape in SAPIO environment and has resulted in the application for a patent. Although IRIS is specialized for the process industry, its domain of application can be extended to other industrial sectors with few changes.

The paper is organized as follows. Sections 2-4 illustrate the new approach and detail the techniques adopted to deploy it in practice. Section 5 shows an example of application. Section 6 concludes the work.

\section{RISK MODELING THROUGH BAYESIAN NETWORKS}

Fault Trees (FTs, Zio (2007)) are widely adopted in quantitative risk analyses performed to verify the fulfillment of legislative and regulatory safety constraints to operate a safety-critical plant. For this reason, they are taken as the basis of the IRIS approach.

FTs are developed by expert risk analysts and used to estimate the probability of occurrence of system failure Top Events (TEs), which are identified as potential events in accident sequences by traditional risk analysis methods (e.g., HAZard and OPerability (HAZOP) analysis and Failure Modes and Effect Analysis (FMEA)), and reported in the plant Risk Assessment Document (RAD).

In IRIS, FTs are first automatically converted into Bayesian Networks (BNs) by a consolidated methodology Khakzad et al. (2013); Bobbio et al. (2001). The reason of converting to BNs is that this allows relaxing some practical limitations of FTs Bobbio et al. (2001):

(1) BNs can easily encode multi-state Basic Events (BEs, e.g., working, slightly deteriorated, strongly deteriorated, etc.) instead of only binary (i.e., working, faulty). This is shown in Section 3.2;

(2) The logical relationships between consequence events and causes, modelled in FT by logic gates such as AND and OR, can be easily modified to accommodate the uncertainty in the event consequence of the combinations of failures Bobbio et al. (2001), see Figure1;

(3) BNs simplify the modeling of functional dependencies among BEs Heckerman et al. (1995), which are typically considered as statistically independent in FT analysis Zio (2007);

(4) BNs help troubleshooting: the field evidences collected from the system can be used to update the conditional probabilities throughout the BNs and infer the most probable cause of failures Jensen and Nielsen (2007).

\begin{tabular}{|c|c|c|}
\hline Traditional AND gate & $B=1$ & $B=0$ \\
\hline$A=1$ & 1 & 0 \\
\hline$A=0$ & 0 & 0 \\
\hline BN AND gate & $B=1$ & $B=0$ \\
\hline$A=1$ & 0.89 & 0.05 \\
\hline$A=0$ & 0.05 & 0.01 \\
\hline
\end{tabular}

Fig. 1.: Example of accommodation of uncertainty in logic gates. Top: fully Boolean truth table of an AND gate. Bottom: uncertain consequence events.

\section{IRIS}

The underlying idea of IRIS is that the overall risk of a process plant depends on key variables, which are represented as BEs in the system FTs. These variable can be classified as Boolean variables, Analog variables, Non-sensored barriers and Behavioral barriers, as detailed in the following subsections.

\subsection{BOOLEAN VARIABLES}

Boolean variables include monitored variables that trigger alarms upon failure detection. An example is the signal monitoring the activation of a pressure switch when over-pressure is detected. An initial occurrence probability is assigned to the $\mathrm{BE}$ of a FT representing a boolean variable. When the $\mathrm{BE}$ occurs, the probability is set to 1 and the system TE probability is updated accordingly.

\subsection{ANALOG VARIABLES}

Analog variables include monitored signals of process variables, such as temperature, pressure, flow. The plant risk increases when the signals of these variables fall out of their nominal ranges, which depend on the plant operational settings. The BN capability of modeling multi-state variables allows easily encoding the analog variables in the risk model. Namely, the range of any monitored signal is discretized in steps (Figure 2 , which are assigned gradual failure probability values depending on their proximity to the failure threshold (see Figure 3 for an example referring to the occurrence of a leakage event). The probability of an analog variable being in each state can be updated in real-time, according to the evidence data collected by the installed sensors. Then, the overall system risk is also automatically updated.

\subsection{NON-SENSORED BARRIERS}

Plant risk also depends on non-sensored components, for which there is no monitoring data to track their health states. For example, consider the Pressure Safety Valve (PSV), which is a dormant 


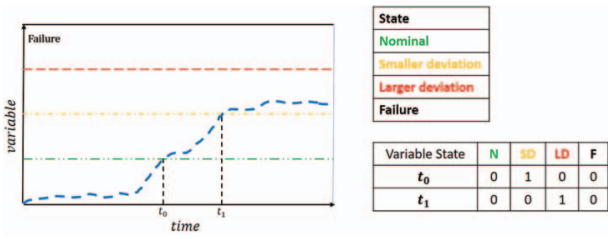

Fig. 2.: Discretization of an analog signal

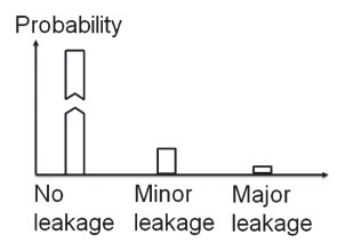

Fig. 3.: Failure probability distribution of a multistate variable. This can be updated over time, based on the proximity to the thresholds reached by the signal

component, whose health state is not known, unless safety checks are performed.

The health state of a non-sensored barrier, however, depends on Influencing Factors (IFs) such as age and maintenance efficiency, which can affect the barrier reliability, quantitatively represented by its failure rate (FR) or "on-demand" response probability.

To consider the contribution to risk of the nonsensored barriers, we link their health states to tracked IFs. Namely, we assume that the inherent reliability behavior relates to the IFs ranging in pre-fixed design conditions. An improvement or a worsening of these conditions changes the barrier reliability (or dually, its failure probability).

To encode in the global risk index the monitoring of the IFs and their effects on the failure probabilities, we use the Multi Attribute Value Theory (MAVT).

\subsubsection{Multi Attribute Value Theory}

MAVT is a systematic approach to address multicriteria decision problems. The key idea is to identify and organize in a hierarchical structure (i.e, the value tree) all the factors influencing the main criterion, which stands at the highest level of the hierarchy.

In IRIS, we consider the Updating Likelihood Factor (ULF) at the highest level, $L_{0}$, of the value tree, which is the quantitative factor used to update the barrier FR.

The hierarchical structure is built depending on the specific barrier. For example, the MAVT hierarchical tree for a PSV is reported in Figure 4. Two main IFs are identified at level $L_{1}$ : "Barrier features" and "Barrier past events", which are further decomposed in their attributes: $\{$ Age, Technology, Operator Technical Evaluation $\}$ and \{Level of Utilization and Maintenance History\}, respectively. These factors are tracked and continuously updated.

For each factor at level $L_{h}$ in the hierarchical structure, the experts qualitatively evaluate the relative importance of its sub-factors at level $L_{h+1}$, translated into importance weights. The sum of the weights of the subfactors at level $L_{h+1}$ of each factor at level $L_{h}$ must sum to 1 .

The method adopted to obtain importance weigths from expert qualitative preference statements is the extension proposed in Salo and Hämäläinen (1995) of the PAIRS method Salo and Hämäläinen (1992). The method allows accommodating imprecise preference statements like "the i-th attribute is more important than the j-th attribute". Finally, all the barriers of the same type in the plant, here referred to as alternatives Punkka and Salo (2013), are assigned a score in the range [0, 100] with respect to every leaf attribute. This score indicates the barrier relevance for the attribute with respect to the best alternative (i.e., score 100) and the worst alternative (i.e., score 0). To obtain the scores for all the alternatives, we apply the SWING method Von Winterfeldt and Edwards (1986). For example, Table 1 shows the extreme conditions of the PSV barrier feature attributes. The best alternative with respect to Age is the new PSV (i.e., age 0), whereas the worst is older than 20 years. These limit values are assigned scores 100 and 0 , respectively. Then, expert judgement is used to evaluate the other alternatives with respects to the limit conditions and with respect to each other Von Winterfeldt and Edwards (1986). For the PSV case, this yields the curve in Figure 5.

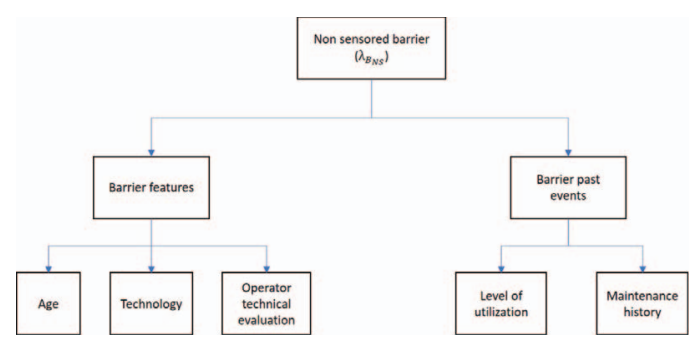

Fig. 4.: Example of Factor Tree for a non sensored barrier

If the mutual preferential independence condition applies Jong and Stone (1976), we can consider the additive function to calculate the overall value $V\left(x_{j}\right)$ of each barrier $x_{j}$ in a bottom-up 
Table 1.: Score limits (SWING method)

\begin{tabular}{lll}
\hline Attribute & Best case & Worst case \\
\hline Age & $<1 \mathrm{y}$ & $>20 \mathrm{y}$ \\
Technology & Innovative & Obsolete \\
Utilization level & Under-stressed & Over-stressed \\
$\ldots$ & $\ldots$ & $\ldots$ \\
\hline
\end{tabular}

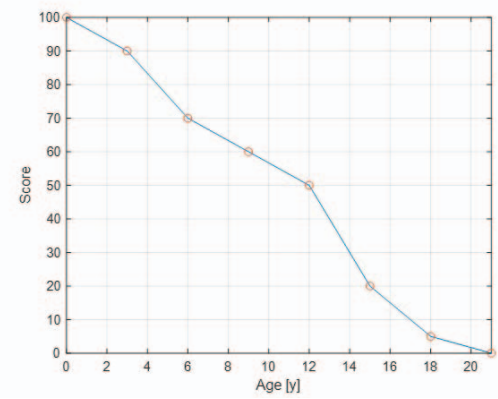

Fig. 5.: Example of score curve for "Age" attribute

logic:

$$
V\left(x_{j}\right)=\sum_{i=1}^{n} w_{i} \cdot v_{i}\left(x_{j}\right)
$$

where $n$ is the number of leaf attributes, $v_{i}\left(x_{j}\right)$ is the score of the alternative $j$ with respect to the $i$-th attribute. $w_{i}$ is the importance weight of the $i$ th attribute with respect to the others, obtained by propagating the weights in each level through the hierarchical tree Jong and Stone (1976); Punkka and Salo (2013); Salo and Hämäläinen (1995).

The value obtained from Eq. 1 for the barrier is mapped into a corrective multiplicative factor of its FR, elicited from experts or inferred from statistical data. Table 2 shows the corrective factors for the PSV. For example, the FR of the worst PSV of the plant is almost double of that of the best PSV.

Table 2.: ULF attribution

\begin{tabular}{ll}
\hline$V_{j}$ & Value \\
\hline$[0-10)$ & 1.3 \\
{$[10-40)$} & 1.1 \\
{$[40-60)$} & 1 \\
{$[60-90)$} & 0.9 \\
{$[90-100]$} & 0.7 \\
\hline
\end{tabular}

\subsection{BEHAVIORAL BARRIERS}

Human factors can strongly contribute to the plant risk. In IRIS, the Cognitive Reliability and Error Analysis Method (CREAM, Hollnagel (1998)) is used to estimate the human reliability, linked to tracked influencing factors such as the time of the day and the expertise of the operators.

CREAM is based on the COntextual COgnitive Model (COCOM), which assumes that any human action description must recognise that it is made in a certain influencing context and that human actions are the result of the smart expertise adapted to circumstance needs, rather than the result of a predetermined sequence of events response Hollnagel (1998). Consequently, COCOM considers expertise and control as two separated aspects of human performance: expertise is a relatively small selection of cognitive functions (human cognition modeling), whereas control describes how experience is implemented. There are four control modes: strategic, tactical, opportunistic and scrambled.

COCOM uses a classification scheme for possible manifestations of erroneous actions and most possible causes. At the highest level of the classification scheme, there is the distinction between effects (phenotypes or manifestations) and causes (genotypes) of the error.

CREAM translates COCOM genotype classification into a set of Common Performance Conditions (CPCs). IRIS considers $N_{C P C}=9 \mathrm{CPCs}$ that are generally used in the literature Hollnagel (1998):

(1) Organization adequacy;

(2) Work condition;

(3) Human-machine interface adequacy and operational support;

(4) Plans/procedures availability;

(5) Number of simultaneous actions;

(6) Available time;

(7) Time of the day;

(8) Training and experience adequacy;

(9) Crew collaborating quality.

Phenotypes are the modes of error of actions. In the CREAM methodology, the error modes are divided into 4 categories: actions at wrong time, actions of wrong types, actions on the wrong object and actions in the wrong place.

CREAM proceeds as shown in Figure 6. Namely, there are two application levels of CREAM: basic and extended. The basic version aims at making a preliminary screening of human interactions with control processes: for every human task, we estimate the Contextual Influence Index (CII), which quantitatively defines the influence of the context on the operator availability. To do this, we identify the number of times the considered CPCs lead to a reduction and/or an improvement of the operative 
performances:

$$
C I I=\sum_{\text {reduced }}-\sum_{\text {increased }}
$$

The CII value allows identifying the actual control mode (among the four identified by COCOM) of the operator in the considered context. The CII values can be also related, with rough estimates, to the operator error probability (Table 3, e.g., Akyuz and Celik (2015)).

Table 3.: Relationship between control modes, CII and human error probability

\begin{tabular}{lll}
\hline Control mode & CII & Human error prob. \\
\hline Strategic & {$[-7,-3]$} & {$[0.5 \mathrm{E}-5,1.0 \mathrm{E}-2]$} \\
Tactical & {$[-3,1]$} & {$[1.0 \mathrm{E}-3,1.0 \mathrm{E}-1]$} \\
Opportunistic & {$[2,5]$} & {$[1 \mathrm{E}-2,0.5]$} \\
Scrambled & {$[6,9]$} & {$[1 \mathrm{E}-1,1]$} \\
\hline
\end{tabular}

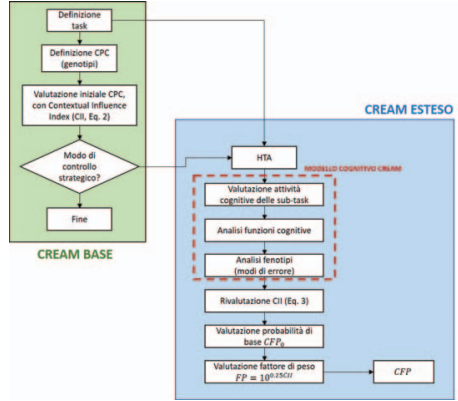

Fig. 6.: Methodology

If the control mode is not strategic, then the extended CREAM is adopted. This is made up of the following steps:

(1) Hierarchical Task Analysis (HTA): the sub-tasks of the task under investigation are examined throug the next steps (2)(5);

(2) Building of the required cognitive profiles. According to the COCOM principles, the cognitive activities linked to every sub-task are individuated and linked to the elementary cognitive functions (Table 4, first column);

(3) Analysis of the phenotypes associated to the elementary cognitive functions. Table 4 reports the CREAM phenotypes and the related Cognitive Failure Probability, $C F P_{0}$, in columns 2 and 3, respectively.
(4) Detailed evaluation of the CPCs. The methodology in Akyuz and Celik (2015) is considered to estimate the Performance Index Indicators (PIIs), which quantify the effect of CPCs on the operator performances (Table 6). This gives the updated value of CII:

$$
C I I=\sum_{i=1}^{N_{C P C}} P I I_{i}
$$

The quality levels of some CPC (e.g., Time of the Day, Training and experience) can be tracked to continuously update their values.

(5) Estimation of the Cognitive Failure Probability (CFP, He et al. (2008)) of the subtask:

$$
C F P=C F P_{0} \cdot 10^{0.25 C I I}
$$

(6) Task failure probability estimation based on the probability of failure of its subtasks. To do this, the rules detailed in Table 5 are adopted.

Table 4.: Cognitive activities, error modes and corresponding nominal probabilties

\begin{tabular}{lll}
\hline Cognitive function Error mode & $C F P_{0}$ \\
Observation & O1: wrong object & $1 \mathrm{E}-3$ \\
& O2: wrong identification & $7 \mathrm{E}-2$ \\
& O3: missed observation & $7 \mathrm{E}-2$ \\
Interpretation & I1: wrong diagnosis & $2 \mathrm{E}-1$ \\
& I2: decisional error & $1 \mathrm{E}-2$ \\
Planning & I3: late interpretation & $1 \mathrm{E}-2$ \\
& P1: prioritization error & $1 \mathrm{E}-2$ \\
Execution & P2: inadequate planning & $1 \mathrm{E}-2$ \\
& E1: wrong action & $3 \mathrm{E}-3$ \\
& E2: wrong action timing & $3 \mathrm{E}-3$ \\
& E3: action on wrong object & $5 \mathrm{E}-4$ \\
& E4: action not planned & $3 \mathrm{E}-3$ \\
& E5: missed action & $3 \mathrm{E}-2$
\end{tabular}

\begin{tabular}{|c|c|c|}
\hline $\begin{array}{l}\text { Task } \\
\text { description }\end{array}$ & $\begin{array}{l}\text { Sub-task } \\
\text { dependence }\end{array}$ & $\begin{array}{l}\text { Task error } \\
\text { probability }\end{array}$ \\
\hline \multirow[t]{2}{*}{ Parallel tasks } & High & $P_{\text {task }}=\min P_{\text {sub-task }}$ \\
\hline & Low/none & $P_{t a s k}=\prod P_{s u b-t a s k}$ \\
\hline \multirow[t]{2}{*}{ Series tasks } & High & $P_{\text {task }}=\max P_{s u b-t a s k}$ \\
\hline & Low/none & $P_{t a s k}=\sum P_{s u b-t a s k}$ \\
\hline
\end{tabular}

Table 5.: Rules to combine the human error probabilities of sub-tasks 
Table 6.: CPC and performance availability

\begin{tabular}{|c|c|c|c|}
\hline $\mathrm{CPC}$ & Qualitative level & Expected effect & PII \\
\hline \multirow{4}{*}{$\begin{array}{l}\text { Organization } \\
\text { adequacy }\end{array}$} & Very efficient & Improved & -0.6 \\
\hline & Efficient & Not significant & 0 \\
\hline & Inefficient & Worsened & 0.6 \\
\hline & Inadequate & Worsened & \\
\hline \multirow[t]{3}{*}{ Work conditions } & Favorable & Improved & -0.6 \\
\hline & Compatible & Not significant & 0 \\
\hline & Incompatible & Worsened & 1 \\
\hline \multirow{4}{*}{$\begin{array}{l}\text { HMI } \\
\text { adequacy }\end{array}$} & Supportive & Improved & -1.2 \\
\hline & Adequate & Not significant & -0.4 \\
\hline & Tollerable & Not significant & 0 \\
\hline & Inappropriate & Worsened & 1.4 \\
\hline \multirow{3}{*}{$\begin{array}{l}\text { Plans/procedures } \\
\text { availability }\end{array}$} & Appropriate & Improved & -1.2 \\
\hline & Accettable & Not significant & 0 \\
\hline & Inappropriate & Worsened & 1.4 \\
\hline \multirow{3}{*}{$\begin{array}{l}\text { Simultaneous } \\
\text { actions }\end{array}$} & Less than capability & Not significant & 0 \\
\hline & Equal to capability & Not significant 0 & \\
\hline & Inappropriate & More than capability & 1.2 \\
\hline \multirow[t]{3}{*}{ Available time } & Adequate & Improved & -1.4 \\
\hline & Temporary inadequate & Not significant & 0 \\
\hline & Continously inadequate & Worsened & 2.4 \\
\hline \multirow[t]{2}{*}{ Time of the day } & Day & Not significant & 1 \\
\hline & Night & Worsened & 0.6 \\
\hline \multirow{3}{*}{$\begin{array}{l}\text { Training and } \\
\text { experience }\end{array}$} & High experience & Improved -1.4 & \\
\hline & Low experience & Not significant & 0 \\
\hline & Inadequate & Worsened & 1.8 \\
\hline \multirow{4}{*}{$\begin{array}{l}\text { Crew } \\
\text { collaboration }\end{array}$} & Very efficient & Improved & -1.4 \\
\hline & Efficient & Not significant & 0 \\
\hline & Inefficient & Not significant & 0 \\
\hline & Inadequate & Worsened & 1.4 \\
\hline
\end{tabular}

\section{IRIS EVALUATION}

In a single process plant, several TEs are built and accepted by the authorities if their occurrence probabilities are smaller than the corresponding acceptability thresholds.

The risk indicator must refer to the risk of the overal plant, which generally includes $f=$ $1, \ldots, F$ production processes.

The risk indicator for the each process can be estimated applying the OR logic to all the related TEs, while properly considering the BEs appearing in multiple FTs. To do this, we have to properly consider the different order of magnitudes of the TE probabilities: the largest ones will prevail in the risk indicator calculation and they may render negligible even sensible increase of the occurrence probabilitites of more rare TEs.

To address this issue, the probability of every TE is partitioned in intervals $I_{i}=\left[p_{i}, \bar{p}^{i}\right], i=1, \ldots, 4$, so that $\bar{p}^{1}$ equals the probability value accepted by the safety authorities to operate the plant.

To communicate the risk to the plant operators, the intervals $I_{i}$ are mapped into intervals $l_{i}=$ $\left[\underline{l}_{i}, \bar{l}_{i}\right] \subseteq[0,10]$, as indicated in Table 7 . In particular, the intervals $l_{i}$ are not equally spaced: the larger length of $l_{1}=[0,5]$ allows emphasizing the small changes of the TE probability due to events not directly resulting in safety consequenses, such as failures of redundant components, delay in maintenance of non-sensored barriers, etc. The resulting sensitivity of the risk index to these events, while still remaining within the first level, is expected to improve the credibility of the index itself by the plant operators.

Table 7.: Residual Risk Indicator intervals

\begin{tabular}{llll}
\hline Level & Name & Range $l_{i}$ & Prob. $I_{i}$ \\
\hline$i=1$ & Normal & {$[0-5]$} & {$[1 e-20,1 e-8]$} \\
$i=2$ & Inspection & {$[5-6.5]$} & {$[1 e-8,1 e-5]$} \\
$i=3$ & Risky & {$[6.5-8]$} & {$[1 e-5,1 e-2]$} \\
$i=4$ & Alert & {$[8-10]$} & {$\left[1 e^{-} 2,1\right]$} \\
\hline
\end{tabular}

We define the IRIS index of the $k$-th TE of the $f$-th process, $f=1, \ldots, F, k=1, \ldots, K^{f}$ as:

$$
\begin{aligned}
& I R I S_{k}^{f}=I R I S_{\text {inf }}+ \\
& \frac{I R I S_{\text {sup }}-I R I S_{\text {inf }}}{\log \left(p_{\text {sup }}\right)-\log \left(p_{\text {inf }}\right)} \cdot\left(\log \left(p_{k}\right)-\log \left(p_{\text {inf }}\right)\right)
\end{aligned}
$$

where:

- $p_{k}$ represents the current estimation of the probability of the $k$-th TE.

- $p_{\text {inf }}=\max _{i}\left\{\underline{p}_{i} \mid \underline{p}_{i} \leq p_{k}\right\}$.

- $p_{\text {sup }}=\min _{i}\left\{\bar{p}_{i} \mid \bar{p}_{i}>p_{k}\right\}$.

- IRIS $S_{i n f}=\underline{l}_{j}, j=\operatorname{argmax}_{i}\left\{\underline{p}_{i} \mid \underline{p}_{i} \leq\right.$ $\left.p_{k}\right\}$.

- $I R I S_{\text {sup }}=\bar{l}_{j}, j=\operatorname{argmin}_{i}\left\{\bar{p}_{i} \mid \bar{p}_{i}>\right.$ $\left.p_{k}\right\}$.

In words, we determine the value of $i \in\{1, \ldots, 4\}$ by comparing $p_{k}$ with the extreme points of the four intevals $I_{i}$. Then, $\log \left(p_{k}\right)$ is rescaled with respect to the orders of magnitude of the extreme points of $I_{i}$ and linearly mapped onto $l_{i}$. Notice that considering the logarithmic scale for the probability allows appreciating very small variations.

The IRIS value for the $f$-th production process, $f=1, . ., F$, is the average of the indices $I R I S_{k}^{f}$, $k=1 . ., K^{f}$ weighted by the corresponding normalized damage $d_{k}^{f} \in[0,1]$ :

$$
I R I S^{f}=\frac{\sum_{k=1}^{K^{f}} I R I S_{k}^{f} \cdot d_{k}^{f}}{\sum_{k=1}^{K^{f}} d_{k}^{f}}
$$

The global IRIS value for the plant is defined as the maximum value among all the $F$ production processes:

$$
I R I S=\max _{f} I R I S^{f}
$$

To justify Eq. 7, on the one hand we can notice that from the reliabilty modeling perspective, the 
$F$ processes are logically combined through an OR gate. According to Levitin and Lisnianski (1998), the score of an OR gate can be estimated as the maximum of the scores of its inputs (see also Compare et al. (2018)). On the other hand, Eq. 7 makes the risk communication clearer. For example, if we consider the sum to combine the risk scores of the $F=4$ processes of the plant considered in the case study, then a risk index $I R I S=7$ can be associated to both $I R I S^{f}=$ $2, f=1, \ldots, 3, I R I S^{4}=1$, and $I R I S^{f}=1$ $f=1, \ldots, 3, I R I S^{4}=4$. These are completely different situations, the latter possibly requiring the emergency escape from the plant.

\section{CASE STUDY}

The SAPIO test plant has $F=4$ production processes. The HAZOP provided $K^{1}=13, K^{2}=3$, $K^{3}=13$ and $K^{4}=4$ TEs, whose probablities have been estimated through the corresponding FTs. These have been translated into BNs.

Figure 7 shows an example of $\mathrm{BN}$ automatically built from the corresponding FT, containing boolean variables, behavioral and non-sensored barriers.

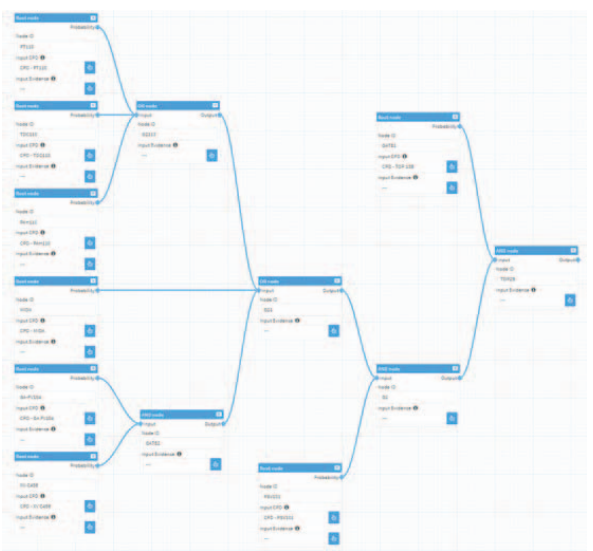

Fig. 7.: Bayesian Network for a Top Event of the first production process

The MAVT approach has been used to estimate the FR of two PSVs (Sections 3.3). Based on interviews with experts, we got the scores in Table 8 , equal for both valves. The importance weights of the attributes of Figure 5 are reported in Table 9. The overall value of the PSV barriers reads:

$$
\begin{aligned}
& V_{P S V}=0.6 \cdot(0.5 \cdot 50+0.25 \cdot 95)+ \\
& 0.4 \cdot(0.6 \cdot 90+0.4 \cdot 90)=78.75
\end{aligned}
$$

Then, according to Table $2, U L F=0.9$ and the PSV FR increases from the nominal value
$\lambda_{P S V, 0}=1.2 \cdot 10^{-2}$ to $\lambda_{P S V}=\lambda_{P S V, 0} \cdot U L F=$ $1.08 \cdot 10^{-2}$. The CREAM procedure has been

Table 8.: Scores for the PSV valves

\begin{tabular}{lll}
\hline Attribute & Value & Score \\
\hline Age & 24 years & 50 \\
Technology & Standard & 90 \\
Other technical evaluation & Light degradation & 95 \\
Utilization level & Lightly used & 90 \\
Maintenance history & Standard & 90
\end{tabular}

Table 9.: Weights assignment

\begin{tabular}{llll}
\hline Attribute $L_{1}$ & Weight & Attributes $L_{2}$ & Weight \\
\hline Barrier features & 0.6 & Age & 0.5 \\
& & Technology & 0.25 \\
& & Other eval. & 0.25 \\
\hline Barrier & 0.6 & Utilization level & 0.6 \\
past events & & Maint. history & 0.4
\end{tabular}

applied to characterize the behavioral barrier related the operator response to the alarm. The CPCs estimation did not yield a strategic control mode, whereby the extended CREAM approach was necessary.

A detailed Hierarchical Task Analysis has been carried out and the cognitive activities linked to each sub-task individuated. Those activities are, then, connected with the elementary cognitive functions, according to the COCOM principles. The subsequent analysis of the phenotypes associated to the elementary cognitive functions has provided the $C F P_{0}$ values (Table 4). The $C I I$ has been evaluated according to Eq. 3 and, then, used to estimate the CFP of each sub-task (Eq. 4), as shown in Table 10. The sub-task dependence

Table 10.: CFP calculation for each sub-task

\begin{tabular}{llllll}
\hline N. & Task step & $\begin{array}{l}\text { Error } \\
\text { mode }\end{array}$ & $C F P_{0}$ & $\begin{array}{l}\text { Weight } \\
\text { factor }\end{array}$ & $C F P$ \\
\hline 0.1 & $\begin{array}{l}\text { Identify and correctly } \\
\text { recognise the alarm }\end{array}$ & $\mathrm{O} 2$ & $7 \mathrm{E}-2$ & 0.282 & $1.974 \mathrm{E}-2$ \\
\hline 0.2 .1 & $\begin{array}{l}\text { Analyse the process } \\
\text { related to the alarm }\end{array}$ & $\mathrm{I} 1$ & $2 \mathrm{E}-1$ & 0.282 & $0.564 \mathrm{E}-1$ \\
\hline 0.2 .2 & $\begin{array}{l}\text { Select the required actions } \\
\text { to solve the alarm }\end{array}$ & I2 & $1 \mathrm{E}-2$ & 0.282 & $0.282 \mathrm{E}-2$ \\
\hline 0.3 & $\begin{array}{l}\text { Conduct the required actions } \\
\text { to solve the problem }\end{array}$ & $\mathrm{I} 2$ & $1 \mathrm{E}-2$ & 0.282 & $0.282 \mathrm{E}-2$ \\
& & & & & \\
\hline
\end{tabular}

is large. Then, the final task probability is the maximum value among the CFPs found in Table 
10. This has led to $P_{\text {task }}=0.0564$, which is almost 50 times larger than the value used in the original HAZOP $\left(P_{\text {task }, 0}=0.001\right)$.

Table 11, first row, shows the effect of the failure of a Pressure Transmitter (PT) on the TE on Figure 7. The other rows show the update of the $I R I S_{f}$ indexes, $f=1, \ldots, F=4$, and the IRIS global index upon the PT failure.

Table 11.: IRIS comparison between nominal and faulty situation

\begin{tabular}{lll}
\hline Variable & Nominal & After PT fault \\
\hline TE 2 posterior prob. & $1 \mathrm{E}-9$ & $1.1 \mathrm{E}-8$ \\
IRIS & 4.9841 & 5.0799 \\
$I R I S_{p p, 2}$ & 4.9656 & 4.9656 \\
$I R I S_{p p, 3}$ & 4.9623 & 4.9623 \\
$I R I S_{p p, 4}$ & 4.9793 & 4.9793 \\
IRIS $S_{\text {global }}$ & 4.9841 & 5.0799
\end{tabular}

\section{CONCLUSIONS}

We have presented the IRIS approach to track risk as a control variable in safety-critical process plants. This produces an overal risk index, which simplifies risk comunication and aids decision making. The next steps for the IRIS development are the development of interfaces with Enterprise Asset Management (EAM) softwares, which allow triggering alarms with Work Requests (WR) and/or Work Orders (WO) for prescriptive maintenance.

\section{References}

Akyuz, E. \& M. Celik (2015). Application of cream human reliability model to cargo loading process of lpg tankers. Journal of Loss Prevention in the Process Industries 34, 39-48.

Bobbio, A., L. Portinale, M. Minichino, \& E. Ciancamerla (2001). Improving the analysis of dependable systems by mapping fault trees into bayesian networks. Reliability Engineering \& System Safety 71(3), 249-260.

Coble, J., P. Ramuhalli, L. J. Bond, J. Hines, \& B. Ipadhyaya (2015). A review of prognostics and health management applications in nuclear power plants. International Journal of prognostics and health management 6, 016.

Compare, M., E. Zio, E. Moroni, G. Portinari, \& T. Zanini (2018). Development of a methodology for systematic analysis of risk reduction by protective measures in tyre production machinery. Safety Science 110, $13-28$.

Daigle, M., I. Roychoudhury, L. Spirkovska, K. Goebel, S. Sankararaman, J. Ossenfort, \& C. Kulkarni $(2017,06)$. Real-time prediction of safety margins in the national airspace.
Di Maio, F., F. Antonello, \& E. Zio (2018). Condition-based probabilistic safety assessment of a spontaneous steam generator tube rupture accident scenario. Nuclear Engineering and Design 326, 41-54.

Drath, R. \& A. Horch (2014). Industrie 4.0: Hit or hype? [industry forum]. IEEE Industrial Electronics Magazine 8, 56-58.

He, X., Y. Wang, Z. Shen, \& X. Huang (2008, 02). A simplified cream prospective quantification process and its application. Reliability Engineering System Safety 93, 298-306.

Heckerman, D., E. H. Mamdani, \& M. P. Wellman (1995). Real-world applications of bayesian networks. Commun. ACM 38, 24-26.

Hermann, M., T. Pentek, \& B. Otto (2016). Design principles for industrie 4.0 scenarios. In 2016 49th Hawaii international conference on system sciences (HICSS), pp. 3928-3937. IEEE.

Hollnagel, E. (1998). Cognitive reliability and error analysis method (CREAM). Elsevier.

Jensen, F. V. \& T. D. Nielsen (2007). Bayesian Networks and Decision Graphs (2nd ed.). Springer Publishing Company, Incorporated.

Jong, N. K. \& P. Stone (1976). Decisions with multiple objectives: Preferences and value tradeoffs. In In Proceedings of the ICML-06 Workshop on Kernel Methods in Reinforcement Learning. Citeseer.

Khakzad, N., F. Khan, \& P. Amyotte (2013). Dynamic safety analysis of process systems by mapping bow-tie into bayesian network. Process Safety and Environmental Protection 91(12), 46-53.

Levitin, G. \& A. Lisnianski (1998). Structure optimization of power system with bridge topology. Electric Power Systems Research 45(3), 201208.

Punkka, A. \& A. Salo (2013). Preference programming with incomplete ordinal information. European Journal of Operational Research 231(1), 141-150.

Salo, A. A. \& R. P. Hämäläinen (1992). Preference assessment by imprecise ratio statements. Operations Research 40(6), 1053-1061.

Salo, A. A. \& R. P. Hämäläinen (1995). Preference programming through approximate ratio comparisons. European Journal of Operational Research 82(3), 458-475.

Von Winterfeldt, D. \& W. Edwards (1986). Decision analysis and behavioral research. Cambridge University Press.

Zio, E. (2007). An introduction to the basics of reliability and risk analysis, Volume 13 . World scientific.

Zio, E. (2018). The future of risk assessment. Reliability Engineering and System Safety 177, 176-190. 